\title{
Influence of Temperature and Pressure on the Free Volume in Thermo-Shrunken Polyethylene by Positron Annihilation
}

\author{
A. DANCH ${ }^{a}, \mathrm{~W} \cdot \mathrm{OSOBA}^{a, *}$ AND J. WAWRYSZCZUK ${ }^{b}$ \\ ${ }^{a}$ Institute of Physics, University of Silesia \\ Uniwersytecka 4, 40-007 Katowice, Poland \\ ${ }^{b}$ Institute of Physics, Maria Curie-Skłodowska University \\ pl. Marii Curie-Skłodowskiej 1, 20-031 Lublin, Poland
}

\begin{abstract}
Positron lifetime measurements were performed as a function of temperature and pressure for thermo-shrunken polyethylene. Mean volume of free volume cavities in the investigated polymer was estimated from the results of the measurements. This mean volume turned out to be the smooth function of temperature (from room temperature to $8.5 \mathrm{~K}$ ) and high pressure (from $0.1 \mathrm{MPa}$ to about $500 \mathrm{MPa}$ ). The effect of trapping of the free electrons produced during positron irradiation was observed at low temperature.
\end{abstract}

PACS numbers: $71.60 .+\mathrm{z}, 78.70 . \mathrm{Bj}$

\section{Introduction}

Positron annihilation spectroscopy turned out to be a very useful method for investigation of different aspects of polymer properties [1,2]. In this paper we present results of the positron lifetime measurements for thermo-shrunken polyethylene as a function of low temperature and high pressure. Both factors influence the fate of positrons in polymers $[3,4]$.

\section{Experimental}

\subsection{Positron lifetime measurements at low temperature}

A conventional, slow-fast coincidence spectrometer with plastic scintillators was used for lifetime measurements as a function of low temperature (Department

${ }^{*}$ corresponding author 
of Nuclear Physics and Its Applications, University of Silesia, Katowice, Poland). The time resolution of the spectrometer, approximated by two Gaussian curves, was determined by analysing the positron lifetimes in kapton foils. Typical values of the full widths at half of the maximum $\left(\mathrm{FWHM}_{i}\right)$ were: $\mathrm{FWHM}_{1}=325.4 \mathrm{ps}$ $\left(I_{1}=90.96 \%\right) ; \mathrm{FWHM}_{2}=473.9$ ps. Positron lifetime spectra were accumulated to approximately $1 \times 10^{6}$ counts. A positron source $\left({ }^{22} \mathrm{Na}\right.$, about $\left.0.8 \div 0.9 \mathrm{MBq}\right)$ was sealed between two kapton foils (about $8 \mu \mathrm{m}$ thick). The measurements were performed in the dark, in vacuum (about $10^{-8} \div 10^{-9}$ mbar at low temperature, and $10^{-4} \div 10^{-5}$ mbar, at room temperature). One spectrum was acquired about $20 \mathrm{~h}$. A closed cycle helium refrigerator system was used to keep the sample at low temperature. A digital microprocessor controlled measurement and a control unit were used to regulate the temperature of the sample. It allowed to keep the temperature of the sample constant, within $\pm 0.030 \mathrm{~K}$. The measurements started at $297 \mathrm{~K}$, and then the sample was cooled down to $8.5 \mathrm{~K}$ and later on it was heated up to $294 \mathrm{~K}$. The measurements were carried out at $40 \mathrm{~K}$ spaced intervals for both the cooling and heating cycles. The sample was kept at the newly chosen temperature for 40 to $50 \mathrm{~min}$ and afterwards the new measurement started.

The sample was made of $1.3 \mathrm{~mm}$ thick sheet of thermo-shrunken polyethylene, from the Institute of Chemistry and Nuclear Technique, Warsaw, Poland. This sheet was made of granules (their density was: $0.933-0.939 \mathrm{~g} / \mathrm{cm}^{3}$ ) of Lupolen 2012D and schwarz 413 (manufactured by BASF) by melting the granules. The sheet was a commercial product. This was the reason that the producer refused to reveal details concerning properties of this product. The thermo-shrunken polyethylene shrinks enormously at about $600 \mathrm{~K}$, this is why it is used, for instance, for electric cables insulation. Authors thought it would be interesting to check its properties at low temperature and high pressure. Four discs shaped pieces (about $12 \mathrm{~mm}$ in diameter) were cut down from the polymer sheet and the positron source was sandwiched between them (two discs on each side of the source). The sample did not undergo any additional thermal treatment before the measurements.

\subsection{Positron lifetime measurements at high pressure}

Positron lifetime measurements as a function of high pressure were performed using a positron lifetime spectrometer (with $\mathrm{BaF}_{2}$ crystals) of Maria CurieSkłodowska University in Lublin, Poland. Time resolution of this spectrometer is approximated by one Gaussian curve. The typical value of the FWHM was 253 ps. The measurements were performed at room temperature $(293 \div 294 \mathrm{~K})$. High pressure (from 0.1 $\mathrm{MPa}$ up to about $500 \mathrm{MPa}$ ) was exerted on the sample by a manually operated hydraulic press SPECAC (P/N 15011/25011). This press allowed to keep the high pressure constant within $\pm 4 \mathrm{MPa}$. The spectra were accumulated till about $1 \times 10^{6}$ counts. One spectrum was acquired about $3 \mathrm{~h}$. The source activity was about $0.2 \mathrm{MBq}$. 


\subsection{Thermal characterization of the samples}

Thermal characterization of the samples by differential scanning calorimetry (DSC) method was performed using Perkin Elmer DSC7 type (the first heating run $(20 \mathrm{deg} / \mathrm{min})$ was recorded). It was found that the change of the specific heat evaluated at the glass transition temperature was $\Delta C_{p}\left(T_{\mathrm{g}}\right)=0.351[\mathrm{~J} /(\mathrm{g} \mathrm{K})]$, the value of the melting temperature: $T_{\mathrm{m}}=379.6[\mathrm{~K}]$, and the melting enthalpy: $\Delta H_{\mathrm{m}}=81.0[\mathrm{~J} / \mathrm{g}]$.

\section{Results and discussion}

The positron lifetime spectra, measured as a function of temperature in the range from about $300 \mathrm{~K}$ to $8.5 \mathrm{~K}$, were analysed using the package programs written by Kirkegaard et al. [5]. Three exponential components were fitted to the measured, raw spectra. The lifetime of the second component, $\tau_{2}$, was fixed. Taking into account our former measurements the value $\tau_{2}=380 \mathrm{ps}$ was taken for this fixed lifetime. Three exponentially decaying components, with no constraints used during numerical analysis, were fitted to measured spectra as a function of high pressure. For these calculations LT program, by Kansy [6], was used. This code was used in this case because the number of channels in these measurements was much larger than in the temperature measurements (the LT code evaluated only discrete values of the positron lifetimes).

The source correction was taken into account during numerical evaluations in both programs. Separate measurements on kapton foils were performed for evaluation of this correction.

According to the model proposed by Tao [7] and Eldrup et al. [8], the longest-lived component of the positron lifetime spectrum may be correlated with the mean radius of the spherical, free volume cavity in the polymer matter. They proposed equation:

$$
\tau_{3}=0.5[1-R /(R+0.1656)+1 /(2 \pi) \sin (2 \pi R /(R+0.1656))]^{-1},
$$

where $\tau_{3}$ is the $o$-Ps lifetime expressed in nanoseconds, $R$ is the mean radius of the spherical well (with an infinitive potential barrier) expressed in $\mathrm{nm}$ and $0.1656 \mathrm{~nm}$ is an empirical constant.

In the presented results the values of $\tau_{3}$ changed from $(2.491 \pm 0.010)$ ns (at about $297 \mathrm{~K})$ to $(1.197 \pm 0.006) \mathrm{ns}($ at $8.5 \mathrm{~K})$ in the temperature measurements and from $(2.463 \pm 0.007) \mathrm{ns}$ (at $0.1 \mathrm{MPa})$ to $(1.249 \pm 0.010) \mathrm{ns}$ (at about $459 \mathrm{MPa}$ ) in the pressure ones.

The value of the volume of the free volume cavities $V$ vs. temperature is shown in Fig. 1. This volume was calculated as the volume of the sphere, where $R$ is taken from Eq. (1).

The $V$ value decreases gradually with decreasing temperature, in the cooling cycle and increases in the heating one. It seems that there is no thermal hysteresis in the presented results. In spite of this, it seems that there is no simple correlation between the relative intensity of the longest-lived component of the 


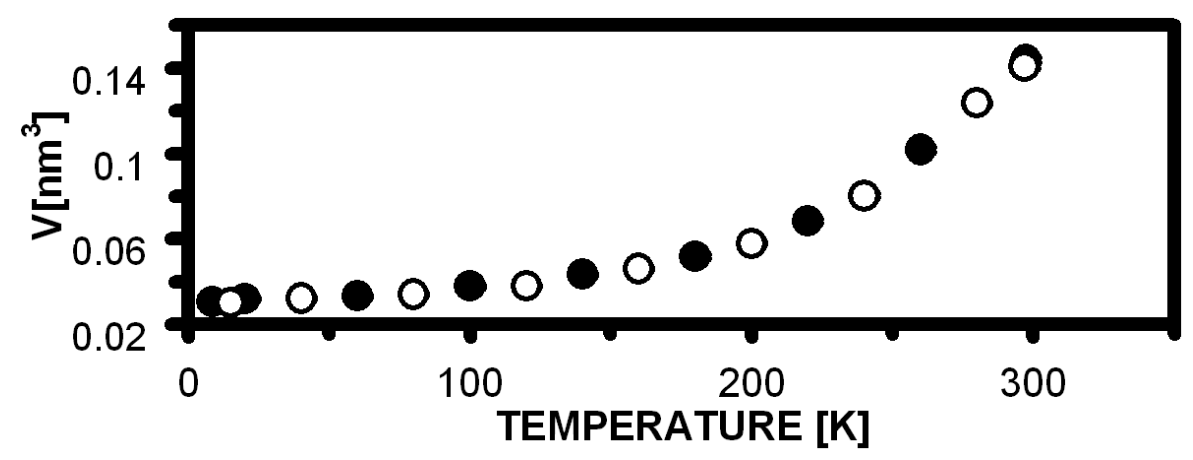

Fig. 1. The values of the volume of the free volume cavities, $V$, vs. temperature: $\bullet-$ cooling cycle, o - heating cycle. Error bars are smaller than dot dimensions.

positron lifetimes, $I_{3}$, and temperature. The $I_{3}$ value as a function of temperature is presented in Fig. 2. It is absolutely clear that the experimental points in the heating cycle do not follow the results observed in the cooling cycle. Going down from room temperature to lower ones, $I_{3}$ decreases (to approximately $200 \mathrm{~K}$ ) and then it starts to gradually increase. Finally, the $I_{3}$ value starts to decrease (in the heating cycle) for temperatures higher than about $120 \mathrm{~K}$.

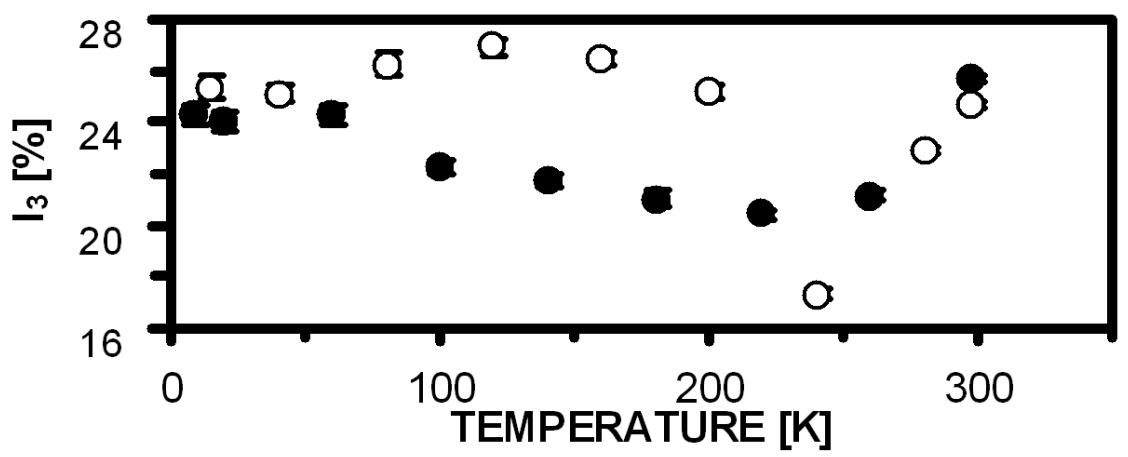

Fig. 2. The $I_{3}$ values vs. temperature: - - cooling cycle, o - heating cycle.

Many authors observed for different polymers qualitatively similar $V$ behaviour vs. temperature, as is presented in this paper: Zrubcová et al. [9], He et al. [10] (their data in the dark, as we performed the experiments in the dark, as well).

The relative intensity of the longest-lived component, $I_{3}$, vs. temperature is more complex. It is well known and generally accepted, in a framework of the spur model of positron annihilation, that a large number of free electrons is produced by positrons in the irradiated sample. When a positron with a kinetic energy of several keV enters an absorber it quickly thermalises. It loses its energy mainly by excitation and ionisation of atoms and molecules of the absorber. The 
electrons released in this process might be trapped in shallow traps (Keyser et al. [11]). According to Hirade et al. [3], free volume cavities may play a role of such traps in the polymer sample. During positron lifetime measurements in polymers, the number of electrons trapped in those traps increases, especially at low temperature. Those electrons are detrapped at higher temperature. If it is so, the increasing number of the trapped electrons will cause the increase in the positronium formation probability. It might result in larger value of the $I_{3}$, during the heating cycle, from $8.5 \mathrm{~K}$ to about $120 \mathrm{~K}$. For higher temperature $I_{3}$ starts to drop as the result of detrapping of the electrons. Figure 2 shows these changes. Characteristic minimum of the $I_{3}$ curve at about $240 \mathrm{~K}$, in heating cycle, was observed also in polyethylene [12] and in polyethylene-co-vinyl acetate [13]. Reiter and Kindl [12] explained the existence of this minimum taking into account the temperature caused contraction of the sample and freezing of local motions of polymers chains.

From Fig. 2 one can find out that $I_{3}$ rises gradually with temperature from about $200 \mathrm{~K}$ to $8.5 \mathrm{~K}$ (in the cooling cycle) and it still rises, in the heating cycle, from $8.5 \mathrm{~K}$ to about $120 \mathrm{~K}$. It seems that $I_{3}$ rises with the elapsed time of the measurements (or with the time when the sample was kept at temperature below $180 \mathrm{~K}) . I_{3}$ starts to decrease after about $250 \mathrm{~h}$ of the measurements. After that time of the measurements the temperature of the sample reached about $120 \mathrm{~K}$ (in the heating cycle). The observed changes of the $I_{3}$ value, with the elapsed time of the measurement, confirms the already mentioned mechanism of the formation of the electron traps in polymers at low temperature. The beginning of the decrease in the $I_{3}$ value at about $120 \mathrm{~K}$ might be explained as the result of detrapping of electrons from their shallow traps, due to molecular motion of the polymer molecules, which results in decrease in the ortho-Ps formation probability. Quite similar behaviour of the longest-lived component of the positron lifetime with time, at low temperature, was observed by Chen et al. [14] and by He et al. [15] (data for the measurements in the dark again).

It was thought that not only low temperature can change the properties of polymers and, as a result of this, parameters of positron annihilation might be changed. The second factor which can disturb the fate of positrons in polymers is high pressure. It was then decided to investigate the influence of high pressure on the $V$ and $I_{3}$ values. The first measurement was accomplished at $0.1 \mathrm{MPa}$ and then the measurements were performed in two cycles: in the first cycle the high pressure increased from about $0.1 \mathrm{MPa}$ to $460 \mathrm{MPa}$. Later on, the pressure decreased to about $0.1 \mathrm{MPa}$. The same sample was used in both cycles. The results of these measurements are presented in Fig. 3 and in Fig. 4. In Fig. 3 the values of the volumes of free volume cavities, $V$, are presented as a function of high pressure. As it was expected, the $V$ values decrease with the increasing pressure. When the high pressure decreases, the $V$ values increase. One can hardly notice any pressure hysteresis in the both cycles. The changes of the $I_{3}$ values with the high 


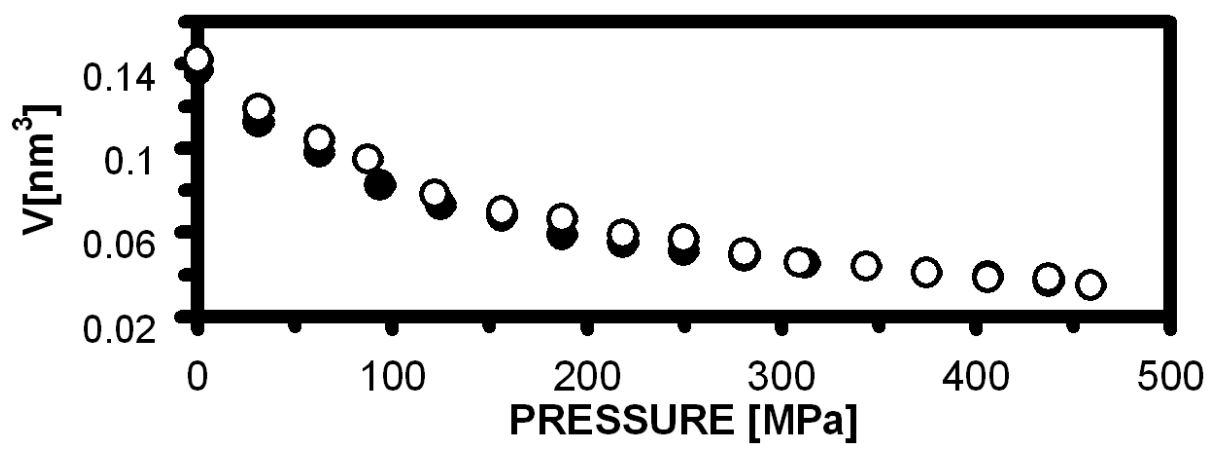

Fig. 3. The values of the volume of the free cavities, $V$, vs. high pressure: $\bullet-$ decreasing pressure, o - increasing pressure. Error bars are smaller than dot dimensions.

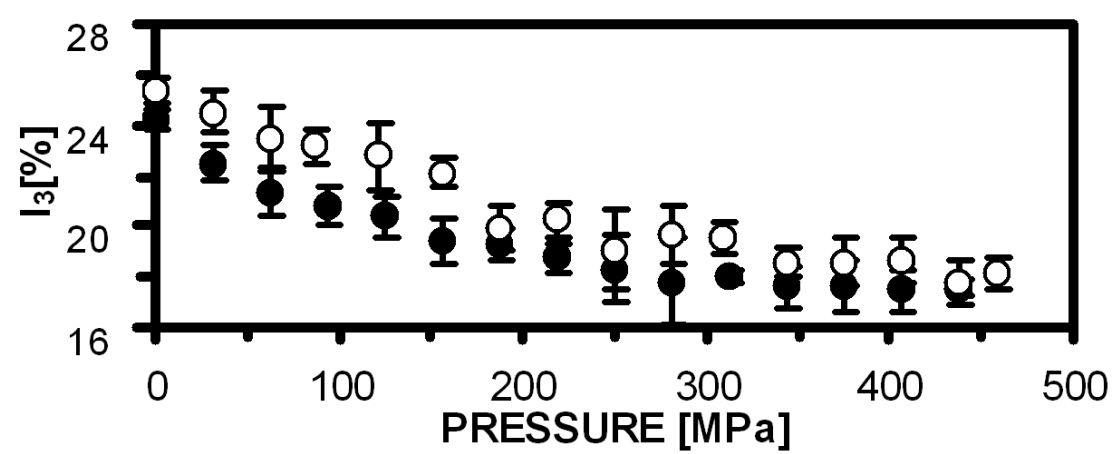

Fig. 4. The $I_{3}$ values vs. high pressure: - - decreasing pressure, ○ — increasing pressure.

pressure are presented in Fig. 4. The $I_{3}$ values are slightly larger in the increasing pressure cycle. The small decrease in the $I_{3}$ values, in the decreasing pressure cycle, is probably observed because the sample did not completely "recover" after the increasing pressure cycle. It might be a kind of a memory effect. This effect needs further investigations.

\section{Conclusions}

The results of the positron lifetime measurements in thermo-shrunken polyethylene as a function of temperature (below room temperature) and high pressure are presented. At low temperature the effect of trapping of the free electrons produced during positron irradiation was observed. Trapping of those electrons in free volume cavities results in the increase in the $o$-Ps formation probability. For the temperature higher than about $120 \mathrm{~K}$, in the heating cycle, those electrons are detrapped and the relative intensity of the longest-lived positron lifetime component, $I_{3}$, decreases. This decrease might be explained by the motion of molecules of the polymer at the temperature higher that about $120 \mathrm{~K}$. The volume 
of the free volume cavities seems to be the smooth function of both factors: temperature and high pressure. The influence of temperature and high pressure on the volume of the free volume cavities, $V$, is similar. This volume decreases gradually with decreasing temperature and increasing pressure. The authors did not notice any thermal or pressure hysteresis in the evaluated volumes of the free volume cavities, $V$, in the performed experiments. To explain the changes of the $I_{3}$ values as a function of high pressure, further investigations are necessary. Probably this is a kind of a memory effect.

\section{References}

[1] Y. Jean, Microchemical J. 42, 72 (1990).

[2] P.E. Mallon, in: Principles and Application of Positron $\&$ Positroniu Chemistry, Eds. Y.C. Jean, P.E. Mallon, D.M. Schrader, World Scientific, New Jersey 2003, p. 253.

[3] T. Hirade, F.H.J. Maurer, M. Eldrup, Radiat. Phys. Chem. 58, 465 (2000).

[4] G. Dlubek, J. Wawryszczuk J. Pionteck, T. Goworek, H. Kaspar, K. Lochhaas, Macromolecules 38, 429 (2005).

[5] P. Kirkegaard, N. Pedersen, M. Eldrup, PATFIT-88: A Data Processing System for Positron Annihilation Spectra on Mainframe and Personal Computers. Ris $\varnothing-$ M-2740, 1 (1989).

[6] J. Kansy, Nucl. Instrum. Methods Phys. Res. A 374, 235 (1996).

[7] S. Tao, J. Chem. Phys. 56, 5499 (1972).

[8] M. Eldrup, D. Lightbody, J. Sherwood, Chem. Phys. 63, 51 (1981).

[9] J. Zrubcová, J. Krištiak, W. Pedersen, N. Pedersen, M. Eldrup, Mater. Sci. Forum 363-365, 359 (2001).

[10] C. He, T. Suzuki, L. Ma, M. Matsuo, V.P. Shantarovich, K. Kondo, Y. Ito, Phys. Lett. A 304, 49 (2002).

[11] R. Keyser, K. Tsuji, F. Williams, in: The Radiation Chemistry of Macromolecules, Ed. M. Mole, Academic Press, New York 1972, Ch. 9.

[12] G. Reiter, P. Kindl, Phys. Status Solidi A 118, 161 (1990).

[13] N. Balcaen, J. de Baerdemaeker, C. Dauwe, K. Cosaert, E. Goethals, F. du Prez, Radiat. Phys. Chem. 58, 497 (2000).

[14] Z. Chen, T. Suzuki, A. Uedono, S. Tanigawa, Y. Ito, Mater. Sci. Forum. 363365, 297 (2001).

[15] C. He, T. Suzuki, L. Ma, M. Matsuo, V.P. Shantarovich, K. Kondo, Y. Ito, Phys. Lett. A 304, 49 (2002). 